\title{
Une nouvelle Stratégie Cybersanté pour la Suisse
}

\author{
Reinhold Sojer \\ Dr, collaborateur scientifique eHealth, $\mathrm{FMH}$
}

La nouvelle Stratégie Cybersanté Suisse est arrivée. Dans un projet en consultation jusqu'au 10 décembre 2017 [1], Confédération et cantons ont mis à jour la Stratégie Cybersanté Suisse de 2007. La stratégie comprend 27 objectifs divisés en trois champs d'action, qui doivent être concrétisés en plusieurs phases entre 2018 et 2022. Les trois champs d'action visent à encourager la numérisation, à la coordonner, et à habiliter professionnels de la santé et patients à la numérisation [2]. Vecteur d'un profond changement structurel, la numérisation touche pratiquement tous les domaines de la société et de l'économie. Par numérisation, on entend généralement la technologisation, c'est-à-dire le recours à de nouvelles technologies de l'information, toujours plus nombreuses à supplanter les moyens analogiques. Un processus qui s'est fortement accéléré

\section{La stratégie comprend 27 objectifs divisés en trois champs d'action, qui doivent être concréti- sés en plusieurs phases entre 2018 et 2022.}

au cours des dernières décennies grâce à la disponibilité de systèmes informatiques performants et à une évolution modérée des coûts. En 1965, Gordon Moore, cofondateur d'Intel, avait prédit que le nombre de circuits intégrés des puces électroniques doublerait tous les douze mois, avant de se raviser dix ans plus tard et de situer cette augmentation exponentielle à 24 mois [3]. Si cette prédiction a dominé l'évolution de la puissance des ordinateurs pendant plusieurs décennies telle une prophétie autoréalisatrice, elle n'a aujourd'hui plus cours notamment en raison du développement du cloud computing (cf. [4]) et de la mise en réseau d'objets intelligents, des appareils hautement spécialisés dotés de capteurs sensoriels qui fonctionnent de manière autonome ou interagissent au sein d'un environnement connecté [5]. Un exemple de cette évolution dans le domaine de la santé est l'application «Streams» du Royal Free de Londres, un système de prise en charge entièrement numérique qui permet de détecter les symptômes d'une insuffisance rénale aiguë à l'aide de technologies connectées mobiles [6].
Comme toute révolution, la révolution numérique a aussi ses victimes. D'une part, la technologisation fait émerger de nouveaux thèmes comme la protection des données ${ }^{1}$, les risques liés à la mise en réseau et à la transparence ou encore les compétences numériques. De l'autre, des processus qui présentent une forte dépendance aux ressources physiques et humaines sont soumis à des changements disruptifs. Nous touchons ici au cœur de toute stratégie de numérisation dans le domaine de la santé. En effet, c'est la transformation numérique, et non la numérisation (technologisation), qui est à l'origine de nouveaux processus et services permettant de résoudre des tâches et des problèmes avec plus d'efficacité. Or le projet de stratégie de la Confédération et des cantons ne tient pas suffisamment compte de cette transformation des processus, comme le démontre la formulation de l'objectif «Remplacement des processus papier par des processus numériques». De même, la transformation a d'abord lieu dans la société avant de pénétrer le monde des affaires en s'adaptant aux besoins des utilisateurs ou des consommateurs des nouvelles technologies, et non l'inverse. La coordination de la numérisation, telle qu'elle figure comme champ d'action dans la stratégie, ne peut donc pas relever de l'Etat. La mission de ce dernier doit consister à promouvoir les innovations et à définir des mesures incitatives là où cela s'avère nécessaire. Il reste à créer des conditions-cadres positives, à placer l'humain au centre de la stratégie du point de vue sociétal et à motiver les utilisateurs professionnels.

\section{C'est la transformation numérique qui est à l'origine de nouveaux processus et services permettant de résoudre des tâches et des problèmes avec plus d'efficacité.}

Même si la Stratégie Cybersanté 1.0 précisait que les personnes, et donc les patients, devaient être au centre d'une telle stratégie, sa mise en œuvre restait très technique. La Stratégie Cybersanté 2.0 est quant à elle placée sous le signe de l'introduction et de la diffusion du dossier électronique du patient (DEP), tout en sou- 


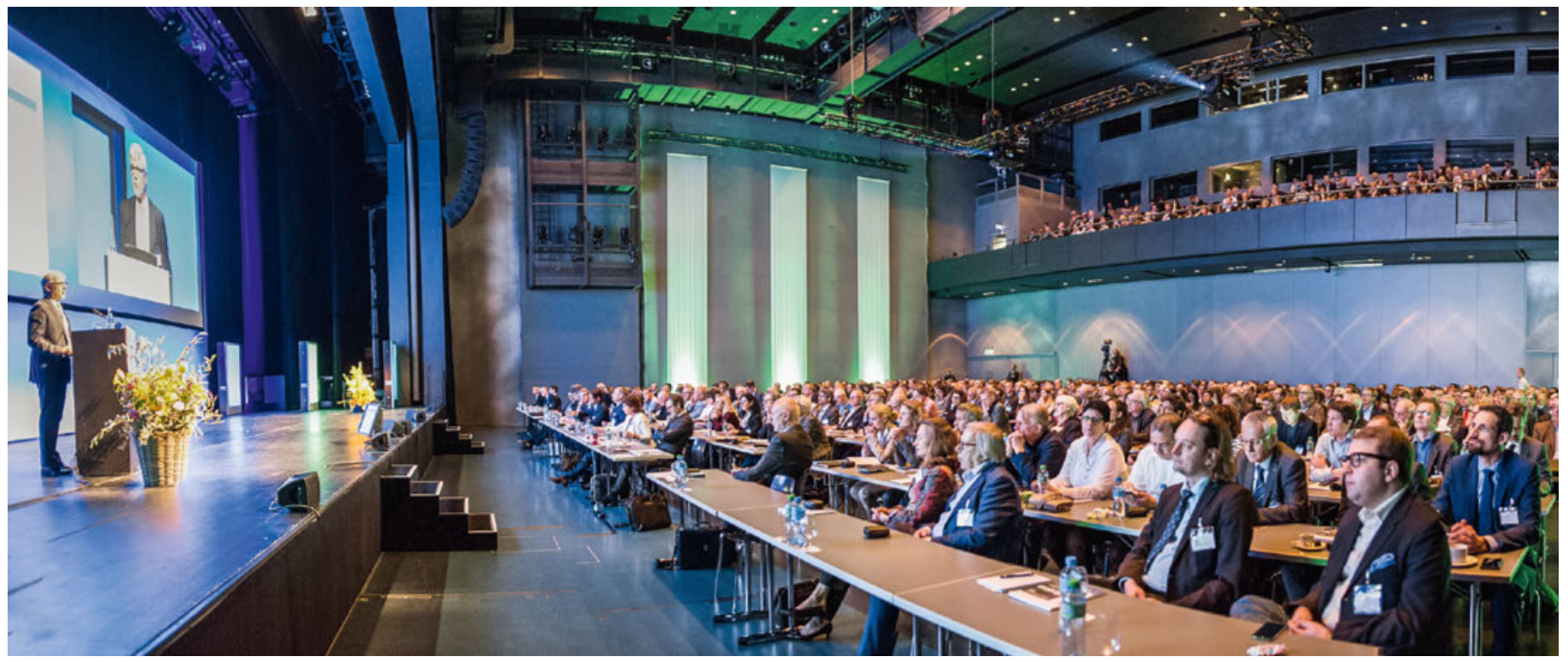

\section{BE SMART - médecine éclairée}

BE SMART - les journées lucernoises des tendances en santé 2018 ont pour thème central la recherche de décisions pertinentes pour une médecine éclairée. Dans le cadre des discussions actuelles qui occupent le monde politique et aussi de plus en plus le grand public, les journées lucernoises entendent apporter une contribution active à un dialogue constructif. Ces journées porteront plus particulièrement sur les questions suivantes: Quels sont les facteurs menant à des soins inappropriés (surtraitement, sous-traitement)? Quelles sont les différences régionales et internationales? Comment y remédier? Faut-il davantage de guides de pratique (contraignants) ou de règlements étatiques? Comment les élaborer? Quels traitements veut-on, à quel prix et avec quels bénéfices? Quels rôles jouent les fournisseurs de prestations, les politiques ou les assureurs? Qu'en est-il de l'éthique et en quoi la numérisation pourrait-elle contribuer à optimiser les processus? En définitive, la question centrale est de savoir quel est le traitement le plus pertinent pour les patients. www.trendtage-gesundheit.ch/fr/ueber uns/Trendtage Gesundheit

lignant la nécessité de nouvelles mesures réglementaires sur des questions comme la santé mobile.

\section{En effet, en plus d'habiliter et de motiver les citoyens, il faut également motiver ceux qui alimenteront le système DEP sans autre compensation financière.}

Lorsque la personne est au centre - promotion des compétences des patients en matière de santé numé-

Correspondance: Dr rer. biol. hum. Reinhold Sojer, Collaborateur scientifique eHealth

FMH Fédération des médecins suisses Elfenstrasse 18 Case postale 300 CH-3000 Berne 15 Tél. 0313591204 reinhold.sojer[at]fmh.ch mais aussi l'intégration des processus du DEP dans le système logiciel des cabinets ne doit présenter aucune faille.

Il y a une dizaine d'années, Martin Denz a montré les conséquences d'une stratégie de cybersanté nationale dans un article du Bulletin des médecins suisses. Il y expliquait notamment, et ses propos sont toujours d'actualité, que la cybersanté est l'expression d'un processus de transformation imminent qui génère de nouveaux processus et de nouveaux services grâce à l'informatisation, mais aussi que les structures et les processus deviennent obsolètes s'ils restent trop longtemps sans être remis en question quant à leur sens, leur utilité et leur nécessité [7].

\section{Crédit photo}

Trendtage Gesundheit Luzern 2017

\section{Références}

1 Organe de coordination cybersanté Confédération-cantons, Stratégie Cybersanté Suisse 2.0 2018-2022 (Projet du 5 septembre 2017), 2017 [online]. Available: https://www.e-health-suisse.ch/ fileadmin/user_upload/Dokumente/2017/F/170911_Entwurf_ Strategie eHealth 2.0 f.pdf [accessed: 10-Dec-2017].

2 Office fédéral de la santé publique, Stratégie Cybersanté (eHealth) Suisse, 2007 [online]. Available: https://www.bag.admin.ch/dam/ bag/fr/dokumente/nat-gesundheitspolitik/ehealth-ch/berichtehealth-strategie-ch.pdf.download.pdf/cybersante-suissestrategie.pdf [accessed: 10-Dec-2017].

3 Moore GE. Cramming more components onto integrated circuit (Reprinted from Electronics, pp. 114-117, April 19, 1965). Proc. Ieee. 1965;86(1):82-5.

4 Kessler T. Sécurité de l'information avec le cloud computing. Bull Méd Suisses. 2017;98(45):1493-4.

5 Waldrop MM. The chips are down for Moore's law. Nature. 2016;530(7589):144-7.

6 Connell A, Montgomery H, Morris S, Nightingale C, Stanley S, Emerson $\mathrm{M}$, et al. Service evaluation of the implementation of a digitally-enabled care pathway for the recognition and management of acute kidney injury. F1000Research. 2017;6:1033.

7 Denz M. Konsequenzen der nationalen E-Health-Strategie. Bull Méd Suisses, 2017;88(9):378-82 (en allemand) 